\title{
MODELAGEM DA CADEIA DE VALOR DO CENTRO DE DESENVOLVIMENTO DE SISTEMAS DO EXÉRCITO BRASILEIRO
}

\section{VALUE CHAIN MODELLING IN THE SYSTEMS DEVELOPMENT CENTER OF THE BRAZILIAN ARMY}

Jonathan Ribeiro Paulino*E-mail: jonathanribeiropaulino@gmail.com

Mariana Mello Pereira* E-mail: pmello.mariana@gmail.com

Mayllon Melo de Miranda* E-mail: mayllon91@gmail.com

Fernanda Moreira Caldas Rodrigues* E-mail: fernandamcrodrigues@gmail.com

Ana Carla Bittencourt Reis* E-mail: anacarlabr@unb.br

*Centro Interdisciplinar de Estudos em Transportes (CEFTRU), Universidade de Brasília (UnB), Brasília, DF

Resumo: A gestão do conhecimento fundamenta-se na compreensão dos processos relevantes para as organizações. É imprescindível que os processos de negócio estejam alinhados com a estratégia organizacional e que a entrega de valor por meio das atividades que geram produtos e serviços seja conhecida. A cadeia de valor identifica essas atividades e as organiza de acordo com o seu foco em agregação de valor, gestão e suporte. A modelagem da cadeia de valor como processo de captura e organização do conhecimento deve ser estudada. Este estudo propõe um modelo de cadeia de valor para o Centro de Desenvolvimento de Sistemas (CDS) do Exército Brasileiro baseada na identificação e análise dos processos da organização. Esta proposta tem como objetivo prover insumos para que a organização coordene e aperfeiçoe a utilização dos seus recursos, garantindo uma gestão do conhecimento efetiva através da compreensão de seus processos e do fluxo de agregação de valor que o modelo apresenta.

Palavras-chave: Gestão do Conhecimento. Cadeia de Valor. Organização Militar. Processos de Negócio. Modelagem.

\begin{abstract}
Knowledge management is based on the understanding of the relevant processes to the organizations. It is essential that the business processes are aligned with the organizational strategy and that the delivery of value by the activities that generate products and services is known. The value chain identifies these activities and organizes them according to their focus on value adding, management and support. The modeling of the value chain as a process of capture and organization of knowledge must be studied. This study proposes a value chain model for the Systems Development Center (CDS) of the Brazilian Army using a modeling methodology based on the discovery and analysis of the organization's processes. This proposal aims to provide input for the organization to coordinate and improve the use of its resources, ensuring an effective knowledge management through the understanding of its processes and the value adding flow that the model presents.
\end{abstract}

Keywords: Knowledge Management. Value Chain. Military Organization. Business Processes. Modelling. 


\section{INTRODUÇÃO}

A crescente competitividade das organizações tem motivado a busca por um melhor desempenho das atividades impulsionando, de maneira significativa, a sua gestão. Essa constante necessidade por aperfeiçoamento produtivo atinge não somente as empresas privadas, mas também o setor público que identifica a necessidade de acompanhar a acelerada mudança econômica, social, cultural e política mundial.

O setor público brasileiro é caracterizado por processos fundamentados na estrutura burocrática, onde há um excesso de regras e procedimentos, o que dificulta e prejudica a sua realização (ZAPELINI, 2011). Entretanto, essa estrutura é também consequência da legislação vigente, que busca garantir a isonomia na realização das atividades.

Com a crescente competitividade, a gestão do conhecimento nas organizações passou a ser um fator preocupante (LEMOS; JOIA, 2012). Grande parte das informações sobre as atividades realizadas são consideradas como conhecimento tácito, inerente aos funcionários da empresa. Isso pode se tornar um grave problema quando o funcionário muda de função ou sai da empresa, além de se tornar um fator prejudicial para o desenvolvimento da organização.

A gestão do conhecimento refere-se à atuação sistematizada, formal e deliberada no sentido de capturar, preservar, compartilhar e (re)utilizar os conhecimentos tácitos e explícitos gerados e aplicados pelos indivíduos durante suas rotinas de trabalho e de melhoria dos processos produtivos, de modo a gerar resultados mensuráveis para a organização e para os indivíduos (TRZESNIAK; MUNIZ; BATISTA, 2009). Assim, o fomento do conhecimento coletivo em uma organização contribui para a competitividade, aumento da capacidade de inovação e da capacidade de resposta (VON KROGH, 1998; HACKBARTH, 1998). Portanto, a gestão do conhecimento nas organizações pretende: (1) tornar o conhecimento explícito e mostrar o seu papel dentro da organização; (2) desenvolver uma cultura intensiva em conhecimento, a qual incentive comportamentos como a busca proativa de conhecimento e o compartilhamento de conhecimento; (3) construir uma infraestrutura de conhecimento, não somente um sistema técnico, mas sim, uma rede 
de ligações entre pessoas com espaço, tempo, ferramentas e encorajamento para interação e colaboração (DAVENPORT; PRUSAK, 1998). Para isso, é preciso considerar a gestão do conhecimento como um processo que envolve, no mínimo, quatro etapas básicas: criação, armazenamento/recuperação, transferência e aplicação de conhecimento. Esses principais processos podem ser subdivididos, por exemplo, na criação de conhecimento interno, aquisição de conhecimento externo, armazenamento do conhecimento em documentos e em rotinas de trabalho, atualização do conhecimento e compartilhamento interno e/ou externo do conhecimento (TEECE, 1998). Esse último, contribui para a resolução de problemas, desenvolvimento de novas ideias e implementação de políticas ou procedimentos (CUMMINGS, 2004; PULAKOS, DORSEY, BORMAN, 2003).

Há uma dificuldade vivenciada pelos órgãos públicos e empresas em garantir uma gestão do conhecimento efetiva. Das várias interpretações sobre gestão do conhecimento, as organizações precisam enfatizar o conhecimento tácito o qual é adquirido pelas experiências dos funcionários e que traz inovações e outras habilidades ocasionando mudança dentro da organização ao ser absorvido. Este conhecimento pode se tornar explícito para ocasionar uma melhora no desempenho dos principais processos da empresa (REIS; MILAN, 2009).

Segundo Lemos e Joia (2012), as organizações estão analisando maneiras de transferir o conhecimento tácito para a empresa e para os demais funcionários. O estudo dos processos, que pode ser promovido pela cadeia de valor, possibilita que o conhecimento esteja disponível para todos os funcionários interessados, além de auxiliar na busca por um melhor desempenho na realização das atividades.

Segundo Porter (1989), a cadeia de valor é uma representação das atividades executadas por toda a empresa para projetar, produzir, comercializar, entregar e sustentar um produto. Kaplinsky e Morris (2001) fazem a definição de cadeia de valor abrangendo o todo, isto é, conjectura uma cadeia de valor global a qual cruza as fronteiras dos países e estabelece elos de valor entre quaisquer empresas envolvidas por algum processo, enquanto que Porter (1989) inclina-se a entender as cadeias de valor dentro das empresas, ou seja, analisa a cadeia de valor corporativa.

Examinar esta cadeia de valor corporativa alerta para como a empresa poderia trabalhar de forma mais eficiente ou mais eficaz (SØRENSEN, 2011), além de 
objetivar seu desempenho financeiro, pois as atividades de valor têm o objetivo de agregar margens aos clientes e, consequentemente, margens de receita e lucro (MACFADYEN et al., 2012).

De acordo com a ABPMP - Association of Business Process Management Professionals - (2013), a cadeia de valor fornece uma visão geral da instituição em processos e em atividades que agregam valor ao produto ou serviço entregue ao cliente. Portanto, uma análise da cadeia de valor determina os processos mais relevantes para a organização e para seu cliente, sendo fundamental aprimorar a execução e garantir a gestão do conhecimento. Onde, processos podem ser definidos como atividades realizadas para atingir um determinado produto, resultado ou serviço pré-determinado (Project Management Institute [PMI], 2008).

Este trabalho tem como objetivo desenvolver a Cadeia de Valor de uma organização militar, listando as atividades finalísticas, as atividades gerenciais e as atividades de apoio desta organização para auxiliar na coordenação e aperfeiçoamento no uso dos seus recursos e na busca de uma gestão do conhecimento efetiva por meio do inter-relacionamento dos processos e atividades presentes na cadeia de valor.

O local objeto do estudo é o Centro de Desenvolvimento de Sistemas (CDS), organização militar diretamente subordinada ao Departamento de Ciência e Tecnologia (DCT) do Exército Brasileiro (EB), "responsável por conceber, gerenciar, desenvolver, integrar e aperfeiçoar sistemas, programas, aplicativos e estruturas físicas e lógicas dos diversos sistemas corporativos e sistemas de informações operacionais do Exército" (CENTRO DE DESENVOLVIMENTO DE SISTEMAS, 2016).

Este artigo é um estudo de caso, aplicado à organização em estudo e está estruturado em cinco partes distintas. A seção 1 apresentou a motivação, o propósito e o objetivo do trabalho. A seção 2 determina os conceitos relacionados a gestão do conhecimento militar. A seção 3 revisa a bibliografia sobre o tema e apresenta as formas de promoção da gestão de conhecimento militar. A seção 4 determina a metodologia adotada na realização do trabalho. A seção 5 apresenta os procedimentos para coleta e análise das informações, bem como os resultados 
encontrados pelos autores. A seção 6 consiste nas considerações finais do trabalho executado, incluindo suas limitações.

\section{GESTÃO DO CONHECIMENTO E SUA RELAÇÃO COM O CONTEXTO MILITAR}

A aplicação dos princípios e técnicas da gestão do conhecimento no contexto militar podem afetar tanto o modo como as novas tecnologias militares são empregadas, quanto a própria evolução da doutrina militar, portanto, é importante determinar como a gestão do conhecimento é definida nesse contexto. A gestão do conhecimento militar refere-se a uma abordagem estratégica que visa alcançar os objetivos de defesa alavancando o valor do conhecimento coletivo através dos processos de criação, coleta, organização, compartilhamento e transferência de conhecimento em ação, de modo a requerer: processos robustos e confiáveis em contextos operacionais; conteúdo e ativos intelectuais focados, precisos, confiáveis, com níveis adequados de recuperação; e processos de criação e conversão de conhecimento compatíveis com o ritmo das operações (MCINTYRE; GAUVIN; WARUSZYNSKI, 2003).

Contudo, antes é preciso entender como o conhecimento é criado. Para isso, existem três principais tipos de conhecimento: (1) tácito: aquele que é compreendido, implícito, informal, enraizado em ações e experiências, difícil de capturar ou compartilhar e existe sem ser declarado, é um conhecimento que não pode ser expresso; (2) explícito: aquele que é apontado em detalhes, formal, não deixa nada meramente implícito, articulado, generalizado e que pode ser manifestado; e (3) implícito: aquele que pode ser expresso, mas não é, pois é pensado como existente nas mentes dos indivíduos (POLANYI, 1967; ALAVI; LEIDNER, 2001).

Com base nas definições supracitadas, é possível entender a criação do conhecimento, a qual ocorre através de um processo em espiral cujo conhecimento é convertido de tácito para explícito em um ciclo contínuo e dinâmico. Sendo assim, a interação destes dois tipos de conhecimento ocorre em quatro estágios de conversão do conhecimento que, segundo Nonaka e Takeuchi (1995) inicia-se com a socialização, onde os indivíduos compartilham experiências e modelos mentais. Esta interação se desenvolve em externalização quando os indivíduos usam metáforas ou analogias para articular o conhecimento tácito oculto. Ao compartilhar e expor tal 
conhecimento ocorre a combinação e, finalmente, quando os indivíduos aprendem, ocorre a internalização do novo conhecimento. $O$ ciclo em espiral recomeça à medida que o conhecimento operacional baseado na experiência de interação aprendida no primeiro ciclo fornece uma base de conhecimento maior para a inovação e crescimento contínuos.

A bem-sucedida gestão do conhecimento no ambiente militar permite ao decisor obter uma compreensão clara da situação das operações dentro do espaço de batalha em relação às forças opostas e ao meio ambiente, bem como visualizar o estado final desejado. Contudo, obter tal cenário é extremamente complexo, pois existem grandes quantidades de informações que requerem extração, triagem e análise. Logo, sem os devidos processos de conversão de conhecimento, pode ocorrer um colapso do conhecimento, portanto, a gestão do conhecimento em ambientes tão complexos é imperativa para a eficácia dos sistemas de conscientização situacional (MCINTYRE; GAUVIN; WARUSZYNSKI, 2003).

A inteligência em situações de defesa nacional exige uma gestão do conhecimento que seja precisa e oportuna para determinar a composição, a posição, as capacidades e as intenções inimigas ou potenciais do inimigo, o que reduz o potencial de surpresa estratégica, operacional, tática ou tecnológica (Canadian Forces Aerospace Warfare Centre [CFAWC], 2007).

Além da conscientização situacional (imagem operacional) e da inteligência numa operação militar, a gestão do conhecimento aplicada ao contexto de defesa nacional também proporciona um alicerce abrangente para a interoperabilidade, treinamento e aquisições.

\section{PRÁTICAS DE GESTÃO DO CONHECIMENTO NO CONTEXTO MILITAR}

Formas de aplicar e melhorar a gestão do conhecimento dentro do contexto militar já vem sendo promovidas através da disseminação da pesquisa em artigos científicos. Sendo assim, uma revisão sistemática da literatura foi realizada no intuito de demonstrar este panorama. As informações foram analisadas para a identificação da forma de promoção da Gestão do Conhecimento no contexto de cada artigo e estão evidenciadas no Quadro 1. 
Quadro 1 - Formas de promoção da Gestão de Conhecimento utilizadas pelos autores

\begin{tabular}{|c|c|}
\hline $\begin{array}{l}\text { Forma de promoção da Gestão de } \\
\text { Conhecimento }\end{array}$ & Autor (es) que utilizaram esta forma \\
\hline $\begin{array}{l}\text { Arquitetura Empresarial para Comando, Controle, } \\
\text { Comunicações, Computadores e Informação } \\
\text { (C4I) Sistema de sistemas (SoS). }\end{array}$ & Mattila (2016). \\
\hline Modelo de comunicação estratégica. & Roca Gonzalez (2015). \\
\hline $\begin{array}{l}\text { Sistema de gerenciamento de conhecimento } \\
\text { (KMS). }\end{array}$ & $\begin{array}{l}\text { Buřita (2015a), Buřita (2015b), Buřita (2014), } \\
\text { Buřita (2012), Velter (2010), Fidock (2010), } \\
\text { Dogan; Henshaw; Urwin (2009), Cosma } \\
\text { (2009). }\end{array}$ \\
\hline Pensamento baseado em efeitos. & Boe (2014). \\
\hline Tecnologias da Informação e Comunicação (TIC). & $\begin{array}{c}\text { Byrne (2013), Ismail; Abdullah (2017), Evans } \\
\text { et al. (2012). }\end{array}$ \\
\hline Ontologia. & $\begin{array}{c}\text { Buřita; Henno; Kiyoki (2012), Buřita et al. } \\
\text { (2011), Ra; Yoo; No (2013), Do; Buřita (2011), } \\
\text { Chen et al. (2011). }\end{array}$ \\
\hline Convergência temporal. & Philp; Martin (2009), Martin; Philp; Hall (2009). \\
\hline Arquitetura da biblioteca digital. & Gao (2008). \\
\hline $\begin{array}{l}\text { Tecnologias de classificação de texto e de } \\
\text { aprendizado de máquina. }\end{array}$ & Lange et al. (2008). \\
\hline Gestão do Conhecimento Ágil (AKM). & Ozturk et al. (2012). \\
\hline $\begin{array}{l}\text { Garantia de Qualidade de Operações de Vôo } \\
\text { Militar (MFOQA). }\end{array}$ & $\begin{array}{l}\text { Marino; Fuller; Walker (2010), Haas et al. } \\
\text { (2008). }\end{array}$ \\
\hline Sistema de gestão de capital intelectual. & Göllner; Mak; Woitsch (2010). \\
\hline
\end{tabular}

Dentre as formas utilizadas, quase que a totalidade envolve algum tipo de tecnologia da informação. Alguns destes autores enfatizam a utilização de uma tecnologia específica para o auxílio na gestão do conhecimento demonstrando sua aplicação, o contexto em que está sendo inserida e como esta foi desenvolvida ou pode ser adquirida. Este é o caso da arquitetura empresarial para C4I SoS, KMS, ontologia, arquitetura da biblioteca digital, tecnologias de classificação de texto e de aprendizado de máquina e sistema de gestão de capital intelectual (BUŘITA et al., 2011, BUŘITA, 2012, BUŘITA; HENNO; KIYOKI, 2012, BUŘITA, 2014, BUŘITA, 2015a, BUŘITA, 2015b, CHEN et al., 2011, COSMA, 2009, DO; BUŘITA, 2011, DOGAN; HENSHAW; URWIN, 2009, FIDOCK, 2010, GAO, 2008, GÖLLNER; MAK; 
WOITSCH, 2010, LANGE et al., 2008, MATTILA, 2016, RA; YOO; NO, 2013, VELTER, 2010). Já outros atores mencionam que a tecnologia da informação auxilia na gestão de conhecimento, sem propor ou aplicar uma em específico. Assim, a TIC e as ontologias são apresentadas como formas de promoção da gestão do conhecimento (BYRNE, 2013, ISMAIL; ABDULLAH, 2017, EVANS et al., 2012).

Entretanto, é importante ressaltar que o sistema de informação tem a capacidade de organizar as informações com base em computação e softwares de apoio à decisão, pela coleta, armazenamento, manipulação e disseminação de dados e informações, para atingir objetivos (STAIR \& REYNOLDS, 2012). É nesse ponto que a cadeia de valor expressa sua contribuição ao proporcionar a expressão das atividades executadas por toda a organização, a fim de entregar valor ao cliente. $O$ conhecimento pode ser considerado como a compreensão de um conjunto de informações necessárias para realização de atividades, ele ajuda a entender as relações na informação (STAIR \& REYNOLDS, 2012).

Nenhum dos autores encontrados utilizam o modelo de cadeia de valor como forma de promoção da gestão do conhecimento. Para que a gestão do conhecimento ocorra, todos da organização são importantes e devem ter consciência da sua contribuição e limitações no cenário em que se encontra, pois esta visa a completa interação entre todos (BAIERLE et al., 2011). Em complemento, Davenport e Prusak (1998) citam que a gestão do conhecimento pretende tornar o conhecimento explícito e mostrar o seu papel dentro da organização.

A cadeia de valor é uma forma de elucidar esta consciência ao transformar conhecimentos tácitos e implícitos em explícito através de seu modelo. A cadeia de valor proporciona um alinhamento entre a estratégia da organização e as atividades realizadas por ela a partir do desdobramento dos macroprocessos identificados em processos e subprocessos, formando uma arquitetura integrada (TORRES et al., 2013), onde existirão atores dominantes que irão liderar a coordenação das atividades e dos outros atores da cadeia de valor.

Grande parte das metodologias de gestão de conhecimento se fundamentam em processo de criação do conhecimento que se baseia em quatro modos de conversão entre conhecimento tácito e explícito. Dois destes modos são a socialização em grupos de trabalho que promove a troca de conhecimentos tácitos e 
a externalização do conhecimento tácito que será codificado, transformando-se em conhecimento explícito (MORENO; SANTOS, 2012). O trabalho de construção da cadeia de valor e sua representação se alinham com estes modos de conversão entre conhecimentos.

\section{METODOLOGIA}

Para o embasamento teórico, foi realizada uma revisão sistemática sobre 0 tema que é abordado pelo artigo. Foram utilizadas as bases de dados Web of Science (WoS) e Scopus como fonte de pesquisa.

Para descrever a situação abordada pelo presente trabalho através da coleta e da análise metodológica das informações, utiliza-se a abordagem investigativa baseada em dados qualitativos do estudo de caso. Yin (2011) reitera a validade do estudo quando objetiva-se analisar eventos contemporâneos os quais o pesquisador tem pouca ou nenhuma interferência sobre a ocorrência, mas que o conhecimento e o entendimento trazem resultados positivos.

O estudo foi desenvolvido no âmbito do Projeto MAPROEx, da Universidade de Brasília (UnB), que tem por objetivo modelar os processos do Exército Brasileiro, bem como fazer o levantamento de requisitos para a modernização do sistema logístico da organização. A equipe do projeto é composta por professores e alunos do curso de Engenharia de Produção e de Engenharia de Software da UnB. A elaboração da Cadeia de Valor é a primeira etapa do projeto, visando a compreensão do fluxo das atividades de valor que serão contempladas pelo sistema logístico em desenvolvimento. A modelagem dos processos é realizada posteriormente e o conhecimento detalhado dos processos apoia a revisão da Cadeia de Valor apresentada neste trabalho.

O presente estudo é uma pesquisa exploratória através de estudo de caso (Yin, 2011), com base no desenvolvimento da cadeia de valor do CDS. Desta forma, o estudo pretende observar aspectos da cadeia de valor que favoreceram a construção do conhecimento a partir das conversões do conhecimento definidas por Nonaka e Takeuchi (1995). 


\section{CADEIA DE VALOR}

O planejamento do trabalho buscou definir as diretrizes para obtenção das informações. Para tanto, o uso de um método replicável é fundamental. Em seguida, a equipe dedicou-se à consolidação e à análise das informações que viabilizaram o desenvolvimento do modelo de Cadeia de Valor.

\subsection{Fase de planejamento}

Verner (2004) apresenta uma metodologia de descoberta de processos que, somados, fazem a composição da Cadeia de Valor das organizações. Essa metodologia foi utilizada como referencial para a condução das reuniões de obtenção de informações. O organograma da organização serviu de base para entendimento de sua estrutura e identificação dos especialistas nos assuntos, militares do CDS, que seriam abordados nas reuniões de obtenção.

De acordo com Verner (2004), a metodologia pode ser classificada quanto à dispersão das sessões, ao sentido da descoberta e à forma de condução. Optou-se pela realização de reuniões distribuídas para melhor acomodar a disponibilidade dos especialistas envolvidos. A identificação dos processos foi realizada segundo 0 organograma da organização partindo da Chefia do CDS, seguindo para os Chefes de Divisão e depois para suas seções e repartições, em sentido top-down. Por fim, a descrição dos processos foi estruturada através de um Formulário de Levantamento de Processos baseado na metodologia SIPOC - Suppliers, Inputs, Process, Outputs and Customers; Fornecedores, Entradas, Processo, Saídas e Clientes, em português - com o objetivo de coletar os insumos para auxílio à construção da Cadeia de Valor.

A partir deste planejamento, as reuniões foram executadas com os atores identificados e consistiu inicialmente em uma introdução explicativa do objetivo e dos benefícios que este trabalho proporciona à organização e em seguida, na coleta das informações, através de questionamentos guiados pelo Formulário, a fim de identificar as atividades realizadas por cada unidade e suas contribuições para o atendimento da missão e agregação de valor para os clientes internos e externos da organização. Todas as informações levantadas foram ratificadas ao final de cada reunião com os próprios atores que as forneceram. 
Com as informações coletadas, seguiu-se para a consolidação e validação dos resultados. A metodologia de consolidação das informações se baseou na aplicação das direções metafóricas de Lankhorst et al. (2009) apresentada na Figura 1, onde um elemento pode ser observado de diferentes pontos de observação para a compreensão de seu posicionamento na cadeia de valor.

Figura 1 - Direções metafóricas para pontos de observação

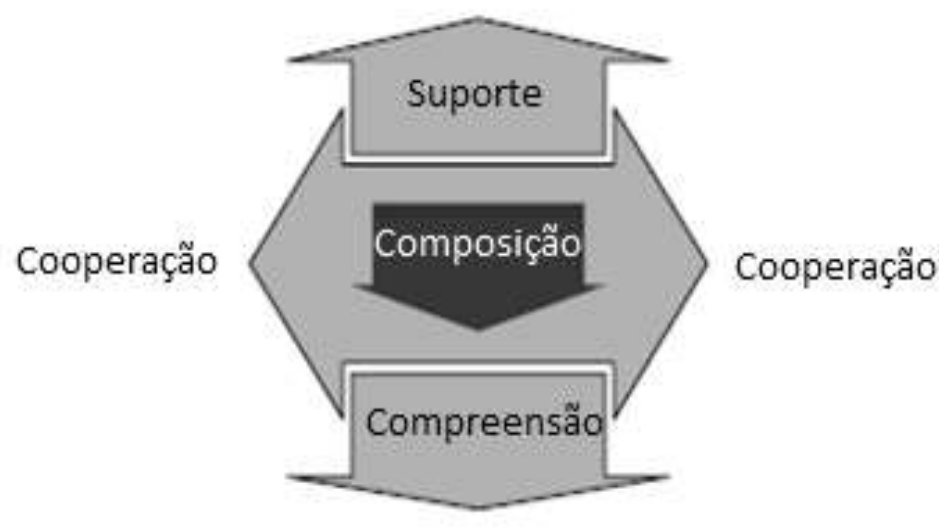

Fonte: Adaptado de Lankhorst et al. (2009)

O elemento sob análise para consolidação das informações pode ser observado em quatro perspectivas:

- $\quad$ Adentro, para composição interna e desdobramento do elemento;

- Para cima, direcionado para os elementos que são suportados;

- Para baixo, direcionado para a sua compreensão por outros elementos;

- Lateralmente, para identificação de elementos pares e em cooperação.

Por fim, Lankhorst et al. (2009) definem as ações de modelagem da Cadeia de Valor que podem ser executadas com cada processo descoberto: introdução do elemento no modelo, refinamento e detalhamento das informações do elemento, abandono de um elemento incompatível, abstração das informações de um elemento, tradução de um elemento para apoiar a compreensão, e documentação das ações tomadas ao longo da consolidação.

A construção da primeira versão da Cadeia de Valor do CDS foi concluída após todos os processos identificados passarem pela análise descrita. Este modelo foi apresentado para validação dos Chefes de Divisão juntamente com o Subchefe e o 
Chefe da organização militar. No total, ocorreram trinta e sete (37) reuniões que foram realizadas em um período de três (3) semanas resultando numa média de doze (12) reuniões por semana realizadas em ambos os turnos.

A consolidação dos resultados se iniciou após o término de todas as reuniões descentralizadas com cada unidade identificada, o que gerou uma listagem de processos. Após a elaboração desta listagem, os processos citados pela Chefia do CDS e pelos Chefes de Divisões foram estudados para dar origem à Cadeia de Valor em um $1^{\circ}$ Nível. Após a definição deste nível, os processos da Chefia do CDS e dos Chefes de Divisão foram lidos novamente para classificar os processos que foram absorvidos pelo $1^{\circ}$ Nível e para agrupar, dentro dos macroprocessos do $1^{\circ}$ Nível, aqueles que ainda não possuíam classificação, surgindo assim o $2^{\circ}$ Nível da Cadeia de Valor. Em seguida, os processos de cada subdivisão foram classificados dentro de cada processo do $2^{\circ}$ Nível e aqueles processos que não conseguiram ser alocados foram estudados para a criação de novos processos de $2^{\circ}$ Nível.

Após toda a classificação dos processos em relação ao nível em que se encontrariam na Cadeia de Valor, uma releitura dos processos foi realizada para readequação, se necessário, dos processos. Com a classificação pronta, os processos começaram a ser refinados por cada macroprocesso do $1^{\circ}$ Nível, do $2^{\circ}$ Nível e do $3^{\circ}$ Nível. Esse refinamento teve como objetivo a leitura dos processos e do breve escopo de cada um constante nos formulários para uma análise de quais seriam os processos finais que comporiam a Cadeia de Valor.

Com o refinamento, os processos começaram a ganhar nomes que abstraíssem a ideia de todos aqueles que estavam sendo contemplados dentro do seu escopo e alguns processos foram eliminados por duplicidade. Por fim, a Cadeia de Valor foi criada e desdobrada em dois níveis que proporcionaram a criação de uma lista de processos, que é insumo principal para a etapa de modelagem dos processos e levantamento de requisitos.

\subsection{Desenvolvimento da cadeia de valor}

A Cadeia de Valor do CDS reúne todas as atividades que agregam valor aos produtos e/ou serviços entregues aos seus clientes a fim de atingir sua missão. Estas atividades podem ser divididas em Macroprocessos Finalísticos, Macroprocessos 
Gerenciais e Macroprocessos de Gestão Interna, conforme a padronização do Escritório de Processos Organizacionais do Exército Brasileiro (EPOEx).

Segundo esta padronização, os Macroprocessos Finalísticos são aqueles que representam a razão da existência da organização, estão diretamente ligados à visão e à missão e agregam valor direto aos clientes desta organização. Os Macroprocessos Gerenciais têm como objetivo garantir a gestão e o alinhamento à estratégia da organização. E os Macroprocessos de Gestão Interna são os responsáveis por suportar, com condições operacionais necessárias, a execução dos processos Finalísticos e Gerenciais (EPOEX, 2015).

Seguindo estes conceitos, a Cadeia de Valor do CDS foi construída, conforme a Figura 2.

Figura 2 - Cadeia de Valor do CDS

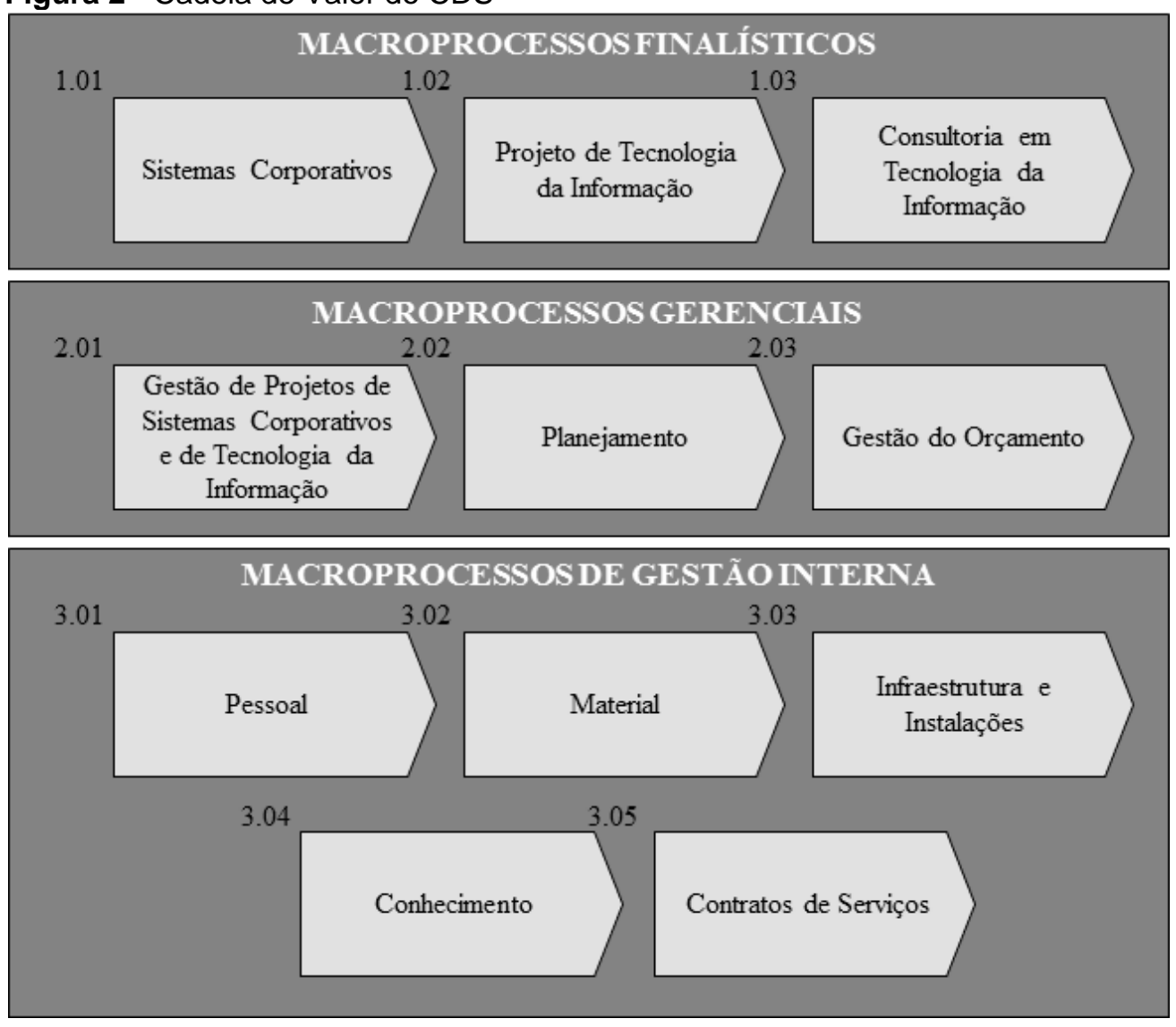

Esta Cadeia de Valor representa o 1ำ Nível da Cadeia de Valor Agregado definido pelo EPOEX (2015) como a representação do mapa estratégico da organização, demonstrando seus principais processos e seu impacto na agregação 
de valor aos produtos e serviços para seus clientes. Este nível foi desdobrado em mais dois níveis, onde se tem o $2^{\circ}$ Nível para todos os macroprocessos do $1^{\circ}$ Nível e só há - $3^{\circ}$ Nível para aqueles macroprocessos do $2^{\circ}$ Nível que ainda podiam ser desdobrados sem que se chegasse a um detalhamento que consistisse na representação de atividades.

O $3^{\circ}$ Nível não foi desdobrado em nenhum outro processo, sendo que seu detalhamento será realizado na própria modelagem de processos através das atividades realizadas para atendimento ao escopo do projeto

Para ilustrar o desdobramento em níveis, segue um exemplo de 2 Nível, conforme a Figura 3, e um exemplo de 3 № Nível, conforme a Figura 4.

Figura 3 - 2ํNível do Macroprocesso Finalístico Sistemas Corporativos

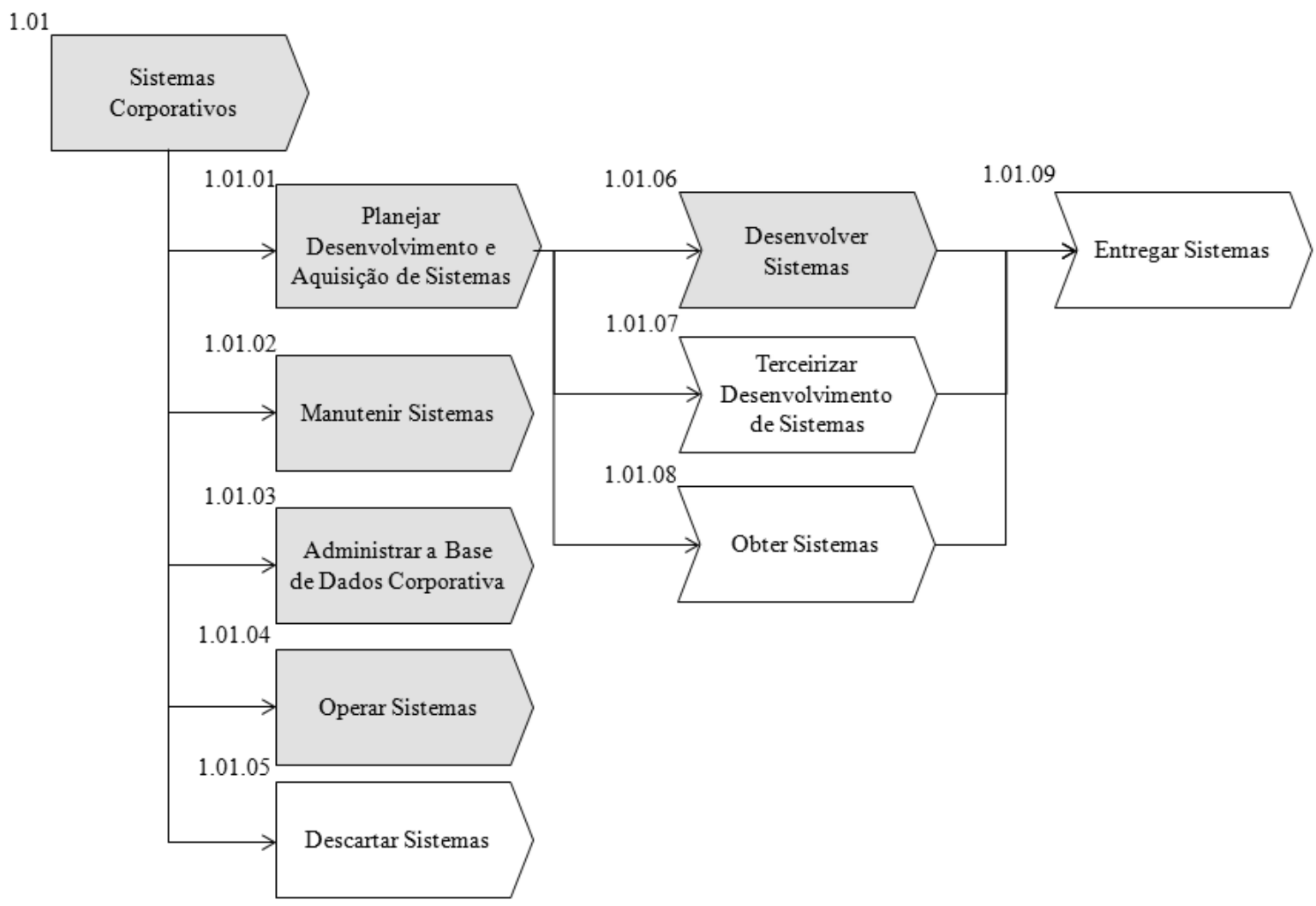

Para uma visualização simples, os elementos que se encontram com preenchimento cinza são elementos que terão seu desdobramento no próximo nível. Já os elementos representados com o preenchimento na cor branca não terão seu desdobramento do próximo nível. 
Figura 4 - 3ํNível do Processo Finalístico Planejar Desenvolvimento e Aquisição de Sistemas 1.01 .01

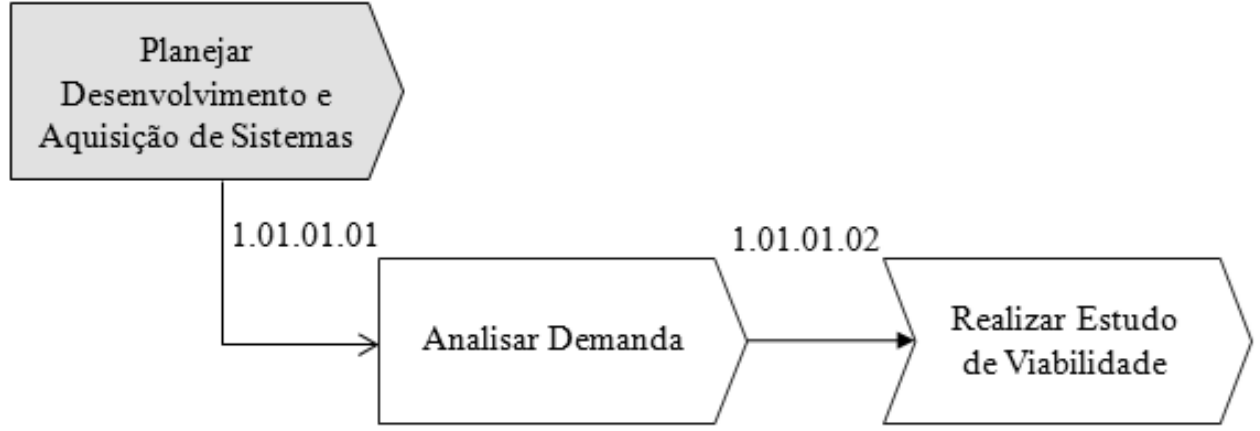

A partir deste desdobramento da Cadeia de Valor foi possível a quantificação inicial de cem (100) processos que compõe a Arquitetura de Processos do CDS e que são relativos aos componentes representados pela coloração branca. Um resumo dos processos pode ser visto no Quadro 2.

\begin{tabular}{|c|c|c|c|}
\hline Classificaçào & Macroprocessos & Qt. de Processos & Total por Classificaçào \\
\hline \multirow{3}{*}{ Finalisticos } & Sistemas Corporatios & 18 & \multirow{3}{*}{30} \\
\hline & Projeto de Tecnologia da Informaçào & 2 & \\
\hline & Consultoria em Tenologia da Informaçào & 10 & \\
\hline \multirow{3}{*}{ Gerenciais } & Gestào de Projetos de Sistemas Corporatiros e de Tecnologia da Informaçào & 4 & \multirow{3}{*}{15} \\
\hline & Planejamento & 4 & \\
\hline & Gestào do Orçamento & $\bar{z}$ & \\
\hline \multirow{5}{*}{ Gestào Interna } & Pessoal & 25 & \multirow{5}{*}{55} \\
\hline & Saterial & 12 & \\
\hline & Intraestrutura e Instalaçòes & 8 & \\
\hline & Conhecimento & $\overline{3}$ & \\
\hline & Contratos de Serriços & 3 & \\
\hline
\end{tabular}

Esta Cadeia de Valor foi validada pela equipe estratégica do CDS e reflete sua missão que consiste na concepção, desenvolvimento, execução e manutenção de Sistemas Corporativos de Tecnologia da Informação e Comunicações Estratégicas; na pesquisa e desenvolvimento de ferramentas de segurança da informação para a proteção e a defesa dos sistemas da Força; e na assessoria aos órgãos da alta administração do Exército Brasileiro nos assuntos relacionados a $\mathrm{Tl}$ e às comunicações estratégicas (CENTRO DE DESENVOLVIMENTO DE SISTEMAS, 2016). 
Esta Cadeia proporciona uma visão das principais atividades desempenhadas pela organização e auxilia em futuras mudanças que possam ocorrer em estruturas da própria organização, assim como na gestão de seus processos.

\section{CONSIDERAÇÕES FINAIS}

O presente trabalho buscou introduzir a modelagem da cadeia de valor como uma forma de promoção da gestão do conhecimento. Ao desenvolver a modelagem dos processos que agregam valor utilizando um método formalizado e sistemático, a captura, organização e preservação do conhecimento geram resultados explícitos para o alinhamento das atividades realizas com a estratégia organizacional. A ação de modelagem permitiu refinar o excesso de dados e a dispersão dos conhecimentos do CDS através da construção metodológica de um modelo com grande potencial de geração de inteligência e vantagem competitiva para organização. Para tanto, houve uma análise minuciosa e um grande esforço de processamento das informações para a construção da Cadeia de Valor.

Uma dificuldade derivada da metodologia de reuniões distribuídas, mencionada também por Verner (2004), foi a captura de fragmentos de processos que são frações de processos ponta a ponta. A fragmentação leva à disparidade de informação e ao entendimento entre as pessoas envolvidas, o que acarreta em maior esforço de compreensão da Cadeia de Valor. Os analistas devem atuar com maior grau de abstração para integrar os fragmentos de processos e ativamente buscar o preenchimento das lacunas de informação. Enquanto isso, os integrantes da organização que deveriam se enxergar representados na consolidação das informações questionam a absorção dos fragmentos de processos em componentes agrupados na Cadeia de Valor. Este problema pode ser tratado ao longo da modelagem de processos, onde a obtenção de informação é aprofundada e maiores considerações podem ser feitas quanto a real composição de processos da organização. Sendo assim, o produto entregue é dinâmico e deve ser atualizado rotineiramente, promovendo um ciclo espiral de conhecimento conforme apontado por Nonaka e Takeuchi (1995).

A repetição das atividades de coleta e processamento das informações que é realizada com a modelagem dos processos torna iterativo o desenvolvimento da 
Cadeia de Valor. A ação posterior é a disseminação do conhecimento, possibilitando o nivelamento e o alinhamento dos envolvidos e consequentemente suporte ao processo decisório, baseado em informação. Assim, podem-se exercer ações de melhoria dos processos que garantam robustez e confiabilidade requerida conforme apontado por Mclntyre, Gauvin e Waruszynski (2003).

Espera-se que os resultados apresentados neste trabalho cheguem aos militares da organização, direcionando-os para o entendimento da importância da gestão do conhecimento organizacional e para a clara compreensão dos objetivos e benefícios que podem ser atingidos com o alinhamento dos processos à estratégia da instituição.

Oportunidades ainda não exploradas como a comparação da Cadeia de Valor produzida com os modelos de referência da área de Tecnologia da Informação tais como Cobit e ITIL serão trabalhadas em próximos estudos. A preocupação com o aprofundamento das análises faz parte do ambiente acadêmico no qual o Projeto MAPROEx está inserido, direcionando os trabalhos à exploração de novas pesquisas e à inovação científica, mantendo-se a atenção ao gerenciamento e à liderança que suportam as ações realizadas, garantindo o sucesso do projeto.

\section{REFERÊNCIAS}

ALAVI, M.; LEIDNER, D. E. Review: Knowledge Management and Knowledge Management Systems: Conceptual Foundations and Research Issues. MIS Quarterly, v. 25, n. 1, p. 107136, 2001. http://dx.doi.org/10.2307/3250961

ASSOCIATION OF BUSINESS PROCESS MANAGEMENT PROFESSIONALS - ABPMP. BPM CBOK: Guia para o Gerenciamento de Processos de Negócio. Versão 3.0. 2013.

BAIERLE, I. C. et al. O ciclo da produção de inteligência como apoio à estratégia de tomada de decisão organizacional. Revista Produção Online. Florianópolis, SC, v.11, n. 4, p. 10861113, out./dez. 2011. http://dx.doi.org/10.14488/1676-1901.v11i4.743

BOE, O. Changing Knowledge Management Strategy in the Norwegian Armed Forces: A Discussion of Effects-Based Thinking as an Alternative Method in the Planning and Execution of Military Joint Operations. Proceeding of Knowledge Management International Conference (KMICe), Malaysia, 12th-15th August, v. 1-2, 2014.

BUŘITA, L. et al. Ontology Development as a Software Engineering Procedure. Digital Information and Communication Technology and Its Applications. BERLIN: Springer Berlin Heidelberg, 2011. p. 1-8. https://doi.org/10.1007/978-3-642-22027-2 1 
BUŘITA, L. The Knowledge Management Systems in the ACR. Croatian Journal

Educational / Hrvatski Casopis za Odgoj I Obrazovanje, v. 14, n.1_supplement, 2012. p.11-19.

BUŘITA, L; HENNO, J.; KIYOKI, Y. Knowledge base modeling and design procedure. Information Modelling and Knowledge Bases XXIII, v. 237. AMSTERDAM: IOS Press, 2012. p. 331-343.

BUŘITA, L. Knowledge Management System for military universities cooperation. In: Communications (COMM), 2014 10th International Conference on. IEEE, 2014. p. 1-4. https://dx.doi.org/10.1109/ICComm.2014.6866667

BUŘITA, L. Portal CEFME for military universities cooperation. In: Military Technologies (ICMT), 2015 International Conference on. IEEE, 2015a. p. 363-367.

https://dx.doi.org/10.1109/MILTECHS.2015.7153665

BUŘITA, L. THE CEFME PORTAL-DISTANCE PREPARATION AND SUPPORT OF THE NATIONAL ADMINISTRATORS. Distance Learning, Simulation And Communication 2015, 2015b. p. 15-20.

BYRNE, B. Knowledge Management in Defence. Proceedings of the 14th European Conference on Knowledge Management (ECKM 2013), v. 1-2, 2013. p. 106-116.

CANADIAN FORCES AEROSPACE WARFARE CENTRE - CFAWC. Out of the Sun: Aerospace Doctrine for the Canadian Forces. Craig Kelman, 2007.

CENTRO DE DESENVOLVIMENTO DE SISTEMAS. Exército Brasileiro. Missão. Disponível em: http://www.cds.eb.mil.br/novo/index.php/editoria-c . s.n.t. Acesso em: 27 de abril de 2016.

COSMA, D. A conceptual framework for the application of QFD to optimize knowledge management in the field of Romanian military education. In: Proceedings of the 10th European Conference on Knowledge Management, v. 1-2. NR READING: Academic Conferences LTD, 2009. p. 917-938.

CUMMINGS, J. N. Work groups, structural diversity, and knowledge sharing in a global organization. Management Science, v. 50, n. 3, p. 352-364, 2004.

https://doi.org/10.1287/mnsc.1030.0134

DAVENPORT, T. H., PRUSAK, L. Working Knowledge: How Organizations Manage What They Know. Harvard Business School Press. Boston: 1998.

DO, P.; BUŘITA, L. Open-source system based on the Ontopia engine. In: Proceedings of the 2011 international conference on applied, numerical and computational mathematics, and Proceedings of the 2011 international conference on Computers, digital communications and computing. World Scientific and Engineering Academy and Society (WSEAS), 2011. p. 138-146.

DOGAN, H.; HENSHAW, M.; URWIN, E. N. A' Soft' Approach to TLM Requirements Capture to Support Through-Life Management. In: Knowledge Science, Engineering and Management (KSEM). BERLIN: Springer Berlin Heidelberg, 2009. p. 458-469. https://doi.org/10.1007/978-3-642-10488-6 44 
EPOEX, 2015. Padrão de Modelagem de Processos. Versão 0.2. 2015.

EVANS, R. D. et al. An investigation into collaboration and knowledge management during product development in the aerospace and defence industry. International Conference on Knowledge Management and Information Sharing, Barcelona, Spain, 4th-7th October, 2012. p. 113-118. https://dx.doi.org/10.5220/0004111001130118

FIDOCK, J. Evaluating information systems: an appropriation perspective. Information Systems Foundations Part Three: the role of design science. CANBERRA ACT: ANU E Press, 2010. p. 121-141.

GAO, L. Knowledge Management-Oriented Architecture Design of Military Equipment Digital Library. In: 2008 IEEE International Symposium on Knowledge Acquisition and Modeling Workshop Proceedings, 2008. KAM Workshop 2008. IEEE International Symposium on, v. 1-2. NEW YORK: IEEE, 2008. p. 960-963. https://dx.doi.org/10.1109/KAMW.2008.4810651

GÖLLNER, J.; MAK, K.; WOITSCH, R. Intellectual capital management using knowledge scorecards: the Austrian national defence academy showcase. Electronic Government and the Information Systems Perspective, p. 249-256, 2010. https://doi.org/10.1007/978-3$\underline{642-15172-9 \quad 23}$

HAAS, D. et al. Using flight data to improve operational readiness in naval aviation. In: ANNUAL FORUM PROCEEDINGS-AMERICAN HELICOPTER SOCIETY. AMERICAN HELICOPTER SOCIETY, INC, 2008. p. 1559.

HACKBARTH, G. The Impact of Organizational Memory on IT Systems. 4th AIS Americas Conference on Information Systems, p. 588-590, agosto 1998.

ISMAIL, M.; ABDULLAH, R. Y. R. Perceptions of knowledge creation, knowledge management processes, technology and applications in military organisations. Malaysian Journal of Library \& Information Science, v. 16, n. 1, p. 73-85, 2017.

KAPLINSKY, R.; MORRIS, M. A handbook for value chain research. Ottawa: IDRC, 2001. Disponível em:

http://asiandrivers.open.ac.uk/documents/Value chain Handbook RKMM Nov 2001.pdf. Acesso em: 28 de abril de 2016.

LANGE, D. S. et al. Text Classification and Machine Learning Support for Requirements Analysis Using Blogs. Innovations for Requirements Analysis: From Stakeholders' Needs to Formal Designs. Berlin: Springer Berlin Heidelberg, 2008. p. 182-195. https://doi.org/10.1007/978-3-540-89778-1 14

LANKHORST, M. et al. Enterprise Architecture at Work: Modelling, Communication and Analysis. 1 ed. Berlim: Springer, 2009.

LEMOS, B.; JOIA, L. A. Fatores relevantes à transferência de conhecimento tácito em organizações: um estudo exploratório. Gestão \& Produção, v.19, n. 233-246, 2012. https://doi.org/10.1590/s0104-530x2012000200001

MACFADYEN, G. et al. Value-chain analysis - An assessment methodology to estimate Egyptian aquaculture sector performance. Aquaculture, v. 362, p. 18-27, 2012. http://dx.doi.org/10.1016/j.aquaculture.2012.05.042 
MARINO, T. A.; FULLER, B. L.; WALKER, J. N. Analysis Techniques for Automatic Flight Maneuver Detection and Evaluation. In: AIAA Infotech@ Aerospace, 2010. p. 3516. https://doi.org/10.2514/6.2010-3516

MARTIN, C. P.; PHILP, W. R.; HALL, W. P. Temporal convergence for knowledge management. Australasian Journal of Information Systems, v. 15, n. 2, 2009. http://dx.doi.org/10.3127/ajis.v15i2.207

MATTILA, J. Military Knowledge Management: Sense-Making, Decision Making and Knowledge Creation. Proceedings of the 17th European Conference on Knowledge Management, 2016. p. 1053-1062.

MCINTYRE, S. G.; GAUVIN M.; WARUSZYNSKI, B. Knowledge Management In The Military Context. Canadian Military Journal, v. 4, n. 1, p. 35-40, 2003.

MORENO, V.; SANTOS, L. H. A. Gestão do conhecimento e redesenho de processos de negócio: proposta de uma metodologia integrada. Perspectivas em Ciência da Informação, v.17, n.1, p. 203-230, jan./mar. 2012. http://dx.doi.org/10.1590/S1413$\underline{99362012000100012}$

NONAKA, I; TAKEUCHI, H. The Knowledge-Creating Company: How Japanese Companies Create the Dynamies of Innovation. New York: Oxford, 1995.

OZTURK, D. et al. Agile knowledge management: A review, reconceptualization, and extension to military applications. In: Annual International Conference of the American Society for Engineering Management 2012, ASEM 2012 - Agile Management: Embracing Change and Uncertainty in Engineering Management, 2012. p. 425-435.

PHILP, W. R; MARTIN, C. P. A philosophical approach to time in military knowledge management. Journal of Knowledge Management, v. 13, n. 1, p. 171-183, 2009. https://doi.org/10.1108/136732709109

POLANYI, M. The tacit dimension. Garden City: Anchor, 1967.

PORTER, M. E. Vantagem competitiva das nações: criando e sustentando um desempenho superior. Campus. Rio de Janeiro, 1989.

PROJECT MANAGEMENT INSTITUTE - PMI. PMBOK: Um Guia do Conhecimento em Gerenciamento de Projetos. 4. ed. Atlanta: PMI, 2008.

PULAKOS, E. D., DORSEY, D. W., BORMAN, W. C. Hiring for knowledge-based competition, in: JACKSON, S. E.; HITT, M. A.; DENISI, A. S. (org): Managing knowledge for sustained competitive advantage: Designing strategies for effective human resource management (p. 155-176). San Francisco: Jossey-Bass. 2003.

RA, M.; YOO, D.; NO, S. Construction and applicability of military ontology for semantic data processing. In: Proceedings of the 3rd International Conference on Web Intelligence, Mining and Semantics. ACM, 2013. p. 15. https://dx.doi.org/10.1145/2479787.2479791

REIS, Z. C.; MILAN, G. S. Gestão do conhecimento - um desafio a ser administrado.

Revista Produção Online, v.9, n.1, p. 73-94, mar. de 2009. http://dx.doi.org/10.14488/1676$\underline{1901 . v 9 i 1.200}$ 
ROCA-GONZALEZ, J. L. Designing dynamical systems for security and defence network knowledge management. a case of study: airport bird control falconers organizations.

Discrete \& Continuous Dynamical Systems - Series S, v. 8, n. 6, 2015. p. 1311-1329. https://dx.doi.org/10.3934/dcdss.2015.8.1311

SØRENSEN, O. J. The Global Value Chain: Formation, Organisation, and Management Perspectives. In: Conference' Global Value Chains and Sustainable Development' 24-25 May 2011. Lyngby: Technical University of Denmark, 2011.

STAIR, R. M. REYNOLDS, G. W. Princípios de Sistemas de Informação. Cegange Learning, 2012.

TEECE, D. Capturing Value from Knowledge Assets: The New Economy, Markets for KnowHow, and Intangible Assets. California Management Review, v. 40, n. 3, p. 55-79, 1998. https://doi.org/10.2307/41165943

TORRES, M. C. G. et al. Cadeia de valor: Os benefícios do alinhamento entre a estratégia governamental e a operacionalização de seus processos. In: CONGRESSO CONSAD DE GESTÃO PÚBLICA, 6, 2013, Brasília.

TRZESNIAK, P.; MUNIZ Jr, J., BATISTA Jr., E. D. Um enunciado definitivo para o conceito de gestão do conhecimento: necessidade para o avanço da ciência e para a aplicação eficaz, in: OLIVEIRA, V. F.; CAVENAGUI, V.; MÁSCULO, F. S. (org): Tópicos Emergentes e Desafios Metodológicos em Engenharia de Produção: Casos, Experiências e Proposições (p. 137-146). Associação Nacional de Engenharia de Produção. Rio de Janeiro: 2009.

VELTER, V. FROM "THE LEARNING ORGANIZATION" TO "THE KNOWLEDGE BASED ORGANIZATION" VIA THE MANAGEMENT OF MILITARY ORGANIZATION. International conference KNOWLEDGE-BASED ORGANIZATION, 2010. p. 657-660.

VERNER, L. The Challenge of Process Discovery. BPM Trends, May, 2004. Disponível em: http://www.bptrends.com/publicationfiles/05-04\%20WP\%20Process\%20Discovery\%20\%20Verner1.pdf. Acesso em: 03 de maio de 2016.

VON KROGH, G. Care in Knowledge Creation. California Management Review, v. 40, n. 3, p. 133-153, 1998. https://doi.org/10.2307/41165947

YIN, Robert K. Applications of case study research. Sage, 2011.

ZAPELINI, M. B. As Big Questions da Administração Pública: Uma Proposta para o Caso Brasileiro. Gestão e Sociedade, v. 5, n. 11, p. 155-175, 2011.

http://dx.doi.org/10.21171/ges.v5i11.1392

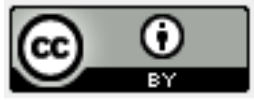

Artigo recebido em 13/02/2017 e aceito para publicação em 01/02/2018 DOI: http://dx.doi.org/10.14488/1676-1901.v18i1.2727 